\title{
LINEAR DIFFERENTIAL EQUATIONS WITH INTERVAL SPECTRUM
}

\author{
RUSSELL A. JOHNSON
}

\begin{abstract}
Let $E$ be the set of equations in the hull of a fixed two-dimensional, almost-periodic, linear differential equation with interval spectrum which admit a bounded solution. Among other things, we prove that $E$ is of first category and of measure zero.
\end{abstract}

1. Introduction. In [8], [9], Sacker and Sell defined the spectrum of a linear, nonautonomous differential system $\dot{x}=B(t) x$. In [2], it was proved that a two-dimensional example of Millionščikov [4] with almost-periodic coefficients has interval spectrum. Our purpose here is to prove several propositions concerning two-dimensional linear systems with uniquely ergodic hull ( 2.1 and 2.2 below) which have interval spectrum. In particular, we prove a result which implies the following statement. Let $\Omega$ denote the hull of the system, let $\mu_{0}$ be the unique ergodic measure on $\Omega$, and let the spectrum of the system be $[-\beta, \beta]$ for some $\beta>0$ (the spectrum may be made symmetric about zero by a simple normalization). Let $\Omega_{0}=\{\omega \in \Omega$ : the equation defined by $\omega$ (see 2.1 and 2.3) admits a bounded solution\}. By the definition of spectrum and [8, Theorem 3], $\Omega_{0}$ is nonempty; we prove that $\mu_{0}\left(\Omega_{0}\right)=0$, and that $\Omega_{0}$ is of first category in $\Omega$.

2. Preliminaries. We introduce notation and review some definitions and results.

2.1. Definitions. Let $C$ be the space of all continuous mappings from $\mathbf{R}$ to the set of $2 \times 2$ real matrices. Give $C$ the topology of uniform convergence on compact sets. The map $\Phi: C \times \mathbf{R} \rightarrow C:(A, t) \rightarrow A_{t}$, where $A_{t}(s)=A(t+s)$, defines a real flow [1] on $C$. Suppose $B \in C$ is uniformly bounded and uniformly continuous. Then $\Omega=\operatorname{cls}\left\{B_{t}: t \in \mathbf{R}\right\} \subset C$ is compact metric, and $\left.\Phi\right|_{\Omega \times \mathbf{R}}$ defines a flow $(\Omega, \mathbf{R})$. We can "extend $B$ to $\Omega$ " as follows: let $b(\omega)=\omega(0)(\omega \in \Omega)$; then $b\left(\omega_{t}\right)=\omega_{t}(0)=$ $\omega(t)(\omega \in \Omega, t \in \mathbf{R})$. In particular, if $\omega_{0} \equiv B \in \Omega$, then $b\left(\omega_{0} \cdot t\right)=B(t)$. We call $\Omega$ the hull of $B$.

2.2. Definitions, notation. Let $(X, \mathbf{R})$ be a flow, where $X$ is a compact metric space. We denote the "position" of $x \in X$ after "time" $t \in \mathbf{R}$ by $x \cdot t$. Say $(X, \mathbf{R})$ is minimal if the orbit $\{x \cdot t: t \in \mathbf{R}\}$ is dense in $X$ for all $x \in X$. Say $(X, \mathbf{R})$ is uniquely ergodic if there is exactly one measure $\mu$ on $X$ which is invariant with respect to $(X, \mathbf{R})$. Here and below, "measure" means "Radon probability measure". See [1], [7].

2.3. Notation. Let $B, \Omega, b$ be as in 2.1 (thus $B$ is a uniformly bounded and uniformly continuous, $2 \times 2$-matrix valued function on $\mathbf{R}$ ).

Received by the editors August 7, 1979.

AMS (MOS) subject classifications (1970). Primary 34C10, 54H20.

(c) 1980 American Mathematical Society 0002-9939/80/0000-0415/\$02.25 
Consider the ODEs

$$
\begin{gathered}
\dot{x}=B(t) x, \\
\dot{x}=b(\omega \cdot t) x \quad(\omega \in \Omega) .
\end{gathered}
$$

We say that equation (1) "induces" equations (1) $)_{\omega}$.

2.4. Definitions. In a well-known way, equations $(1)_{\omega}$ induce a flow (a linearskew-product flow, or LSPF) on $\Omega \times \mathbf{R}^{2}$ [8], [10]. Let $L$ denote this LSPF. The spectrum of $L, \operatorname{Sp}(L)$, is defined to be $\{\lambda \in \mathbf{R}$ : the equation $\dot{x}=(-\lambda I+b(\omega \cdot t)) x$ admits a nonzero bounded solution for some $\omega \in \Omega$ \}; see [8].

As a consequence of the Sacker-Sell spectral theorem [9, Theorem 2], and techniques of [2], we have the following.

2.5. Proposition. Let $L$ be the LSPF of 2.4. Suppose $(\Omega, \mathbf{R})$ is uniquely ergodic. Then $\operatorname{Sp}(L)$ is (i) a single point or (ii) two points or (iii) a nondegenerate closed interval.

2.6. Definitions. Let $L$ be the LSPF of 2.4. Let $\mathbf{K} \subset \mathbf{R}^{2}$ be the unit circle, and let $P$ be projective 1-space. Since $L$ is linear, it induces flows on $\Omega \times K$ and on $\Sigma=\Omega \times \mathbf{P}$; these are defined as follows. If $\left(\omega, x_{0}\right) \in \Omega \times \mathbf{K}$, and if $x(t)$ is the solution to $(1)_{\omega}$ such that $x(0)=x_{0}$, then $\left(\omega, x_{0}\right) \cdot t=(\omega \cdot t, x(t) /\|x(t)\|)$. If $(\omega, l)$ $\in \Sigma$ (thus $l$ is a line through the origin in $\mathbf{R}^{2}$ ), and if $x_{0}$ is a nonzero vector in $l$, then $(\omega, l) \cdot t=(\omega \cdot t, l(t))$, where $l(t)$ is the line containing $x(t)$, and $x(t)$ solves $(1)_{\omega}$ with $x(0)=x_{0}$. Let $\pi_{p}: \Sigma=\Omega \times \mathbf{P} \rightarrow \Omega$ be the projection onto the first factor.

2.7. Definitions. Let $\theta$ be the (usual) angular coordinate on $K$, and let $\varphi=2 \theta$ be the corresponding coordinate on $\mathbf{P}^{\mathbf{1}}$. Let us write

$$
b(\omega)=\left[\begin{array}{ll}
a(\omega) & -c(\omega) \\
c(\omega) & a(\omega)
\end{array}\right]+\left[\begin{array}{ll}
\delta(\omega) & \varepsilon(\omega) \\
\varepsilon(\omega) & -\delta(\omega)
\end{array}\right]
$$

In polar coordinates $(r, \theta)$, equations $(1)_{\omega}$ become

$$
\begin{gathered}
\dot{\theta}=c(\omega \cdot t)+\varepsilon(\omega \cdot t) \cos 2 \theta(t)-\delta(\omega \cdot t) \sin 2 \theta(t) \\
\frac{d}{d t} \ln r(t)=a(\omega \cdot t)+\delta(\omega \cdot t) \cos 2 \theta(t)+\varepsilon(\omega \cdot t) \sin 2 \theta(t) .
\end{gathered}
$$

From $(2)_{\omega}$, the flow $(\Sigma, \mathbf{R})$ is independent of $a(\omega)=\frac{1}{2} \operatorname{tr} b(\omega)$. Since our results will depend only on properties of this flow, we will assume tr $b(\omega) \equiv 0$.

2.8. Remark. Assume tr $b(\omega) \equiv 0$, and define

$$
f_{*}: \Sigma \rightarrow \mathbf{R}: f_{*}(\omega, \varphi)=\delta(\omega) \cos \varphi+\varepsilon(\omega) \sin \varphi
$$

By (3) $\omega_{\omega}$ and (4), we have

$$
\frac{1}{t} \ln \frac{\|x(t)\|}{\|x(0)\|}=\frac{1}{t} \int_{0}^{t} f_{*}((\omega, \varphi) \cdot s) d s
$$

whenever $x(t)$ is a solution to $(1)_{\omega}$ such that the ray defined by $x(0) \neq 0$ has coordinate $\varphi$.

From 2.5 and techniques and results of $[2, \S 3]$, we have 
2.9. Theorem. Suppose $(\Omega, \mathbf{R})$ is uniquely ergodic, and suppose $\operatorname{tr} b(\omega)=2 a(\omega) \equiv$ 0. Assume also that $\operatorname{Sp}(L)$ is a nondegenerate interval. Then $\operatorname{Sp}(L)=[-\beta, \beta]$ for some $\beta>0$. There are exactly two ergodic measures, $\mu_{1}$ and $\mu_{2}$ on $\Sigma$, and $\int_{\Sigma} f_{*} d \mu_{1}$ $=-\beta, \int_{\Sigma} f_{*} d \mu_{2}=\beta$ (or vice versa). There are disjoint Borel sets $B_{1}, B_{2} \subset \Sigma$ such that $\mu_{i}\left(B_{i}\right)=1$, and $\operatorname{card}\left(B_{i} \cap \pi_{p}^{-1}(\omega)\right)=1$ for $\mu_{0}$-a.a. $\omega \in \Omega(i=1,2)$. Here $\mu_{0}$ is the unique invariant measure on $\Omega$.

\section{Results.}

3.1. Assumptions, NOtation. Notation $\left(B, b, \Omega, \Sigma, L, f_{*}\right)$ is as in $\S 2$. We assume $\operatorname{tr} b(\omega) \equiv 0$, and that $(\Omega, \mathbf{R})$ : (i) is minimal; (ii) admits a unique invariant measure $\mu_{0}$. We assume $\operatorname{Sp}(L)$ is a nondegenerate interval; thus $\operatorname{Sp}(L)=[-\beta, \beta]$ for some $\beta>0$.

3.2. Remark. If $\operatorname{tr} b(\omega)=2 a(\omega)$ is not identically zero, and if $\operatorname{Sp}(L)$ is a nondegenerate interval, then $\operatorname{Sp}(L)=\left[a_{0}-\beta, a_{0}+\beta\right]$, where: (i) $a_{0}=$ $\int_{\Omega} a(\omega) d \mu_{0}(\omega)$; (ii) $[-\beta, \beta]$ is the spectrum of the LSPF obtained from the equations $\dot{x}=[b(\omega \cdot t)-a(\omega \cdot t) I] x$.

The proposition below has been stated in the literature [9]; we give a proof.

\subsection{Proposition. The flow $(\Sigma, \mathbf{R})$ contains a unique minimal subflow $(M, \mathbf{R})$.}

Proof. The flow $(\Sigma, \mathbf{R})$ contains at least one minimal subflow [1]. Suppose $M_{1}$ and $M_{2}$ are disjoint minimal subsets of $\Sigma$. Let $\mu_{1}$ and $\mu_{2}$ be the two measures on $\Sigma$ which are ergodic with respect to $(\Sigma, \mathbf{R})(2.9)$. Since each $\left(M_{i}, \mathbf{R}\right)$ admits an ergodic measure [7], we must have, say, $\mu_{i}\left(M_{i}\right)=1(i=1,2)$. Then $\left(M_{1}, \mathbf{R}\right)$ and $\left(M_{2}, \mathbf{R}\right)$ are uniquely ergodic, hence by 2.9 and [7, pp. 498-511], we have

$$
\lim _{|t| \rightarrow \infty} \frac{1}{t} \int_{0}^{t} f_{*}((\omega, \varphi) \cdot s) d s=-\beta \quad \text { resp. } \beta
$$

for all $(\omega, \varphi) \in M_{1}$ resp. $M_{2}$.

Recall now that $\pi_{p}: \Sigma \rightarrow \Omega$ is the projection. Since $(\Omega, \mathbf{R})$ is minimal, $\pi_{p}\left(M_{1}\right)=\Omega$ $=\pi_{p}\left(M_{2}\right)$. It follows that zero cannot be in the spectrum of $L$. For, if it were, some equation (1) $\omega_{\omega_{0}}$ would have a nonzero bounded solution $x_{0}(t)(2.4)$. Let $\left(\omega_{0}, \varphi_{1}\right) \in$ $M_{1}$, and let $\tilde{x}_{1} \neq 0$ be on the line in $\mathbf{R}^{2}$ defined by $\varphi_{1} \in \mathbf{P}^{1}$. If $x_{1}(t)$ satisfies $(1)_{\omega_{0}}$ with $x_{1}(0)=\tilde{x}_{1}$, let $\Psi(t)$ be the fundamental matrix formed from $x_{0}(t)$ and $x_{1}(t)$ $\left(x_{0}(t)\right.$ and $x_{1}(t)$ are linearly independent because, by (*), $x_{1}(t)$ cannot be bounded as $t \rightarrow \infty)$. Since $\operatorname{tr} b(\omega) \equiv 0$, Liouville's formula implies that $\operatorname{det} \Psi(t)=$ const. But, by (*), det $\Psi(t) \rightarrow 0$ as $t \rightarrow-\infty$. This contradiction implies that zero is not in $\operatorname{Sp}(L)$.

Now, by $2.9, \operatorname{Sp}(L)$ must be a point or two points. This contradicts our assumption (3.1). The proof is completed.

3.4. Remark. If we combine the Birkhoff ergodic theorem [7] with 2.9, we see that there is a set $\Omega_{1} \subset \Omega$ of $\mu_{0}$-measure 1 such that, if $\omega \in \Omega_{1}$, then (1) has two solutions, $x_{1}(t)$ and $x_{2}(t)$, satisfying

$$
\lim _{|t| \rightarrow \infty} \frac{1}{t} \ln \left\|x_{1}(t)\right\|=\beta, \quad \lim _{|t| \rightarrow \infty} \frac{1}{t} \ln \left\|x_{2}(t)\right\|=-\beta .
$$


Thus, in a measure-theoretic sense, "most" equations (1) $)_{\omega}$ have solutions with "regular" asymptotic behavior. This should be contrasted with the following result.

3.5. ThEOREM. There is a residual set $\Omega_{2} \subset \Omega$ (i.e., a set containing a dense $G_{\delta}$ ) such that, if $\omega \in \Omega_{2}$, then (1) $)_{\omega}$ has a solution $x_{\omega}(t)$ satisfying

$$
\left(\varlimsup_{t \rightarrow \infty}, \underset{t \rightarrow \infty}{\lim }, \varlimsup_{t \rightarrow-\infty}, \underset{t \rightarrow-\infty}{\lim }\right) \frac{1}{|t|} \ln \left\|x_{\omega}(t)\right\|=(\beta,-\beta, \beta,-\beta) .
$$

Here $\beta$ is defined by $\operatorname{Sp}(L)=[-\beta, \beta]$.

Proof. Consider the function $f_{*}$ of 2.8. Let $\mu_{1}$ and $\mu_{2}$ be the two ergodic measures on $\Sigma$. If $M$ is the unique minimal subset of $\Sigma$, then $\mu_{1}(M)=1=\mu_{2}(M)$. By 2.9, we may assume that $\int_{M} f_{*} d \mu_{1}=\beta>0$, and that $\int_{M} f_{*} d \mu_{2}=-\beta<0$. By [3, Theorem 4.3], there is an invariant, residual subset $M_{2} \subset M$ such that, if $(\omega, \varphi) \in M_{2}$, then

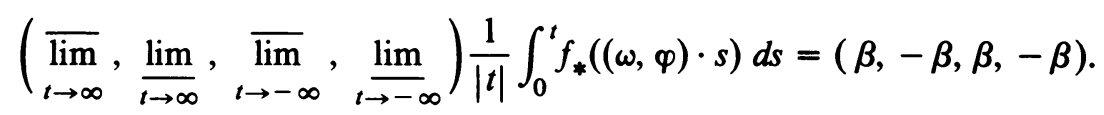

By (5), each such $(\omega, \varphi)$ gives rise to a solution $x_{\omega}(t)$ with the properties described in 3.5.

To complete the proof, note that [11, Lemma 3.1] informs us that, if $\Omega_{2}=$ $\pi_{p}\left(M_{2}\right)$, then $\Omega_{2}$ is a residual subset of $\Omega$.

3.6. REMARKS. (a) The previous result extends (in the two-dimensional case) Theorem 5 of [9], according to which residually many equations (1) $\omega_{\omega}$ admit solutions $x(t)$ satisfying $\left(\overline{\lim }_{t \rightarrow \infty}, \varlimsup_{t \rightarrow-\infty}\right)(1 /|t|) \ln \|x(t)\|=\beta$. Our Theorem 3.5 appears to follow from neither the statement nor the proof of this result of [9].

(b) According to results of Millionščikov [6], every nonzero solution of (1) $(\omega \in \Omega)$ satisfies

$$
\left(\varlimsup_{|t-s| \rightarrow \infty}, \underset{|t-s| \rightarrow \infty}{\lim }\right) \frac{1}{|t-s|} \ln \frac{\|x(t)\|}{\|x(s)\|}=(\beta,-\beta) .
$$

This can be proved using 3.5.

Our final theorem implies the result discussed in the Introduction.

3.7. TheOREM. Given $\alpha \in \operatorname{Sp}(L)=[-\beta, \beta]$, let $\Omega_{\alpha}=\{\omega \in \Omega \text { : equation (1) })_{\omega}$ admits a solution $x_{\alpha}(t)$ such that $e^{-\alpha t}\left\|x_{\alpha}(t)\right\|$ is bounded on $\left.-\infty<t<\infty\right\}$. By 2.4, $\Omega_{\alpha} \neq \varnothing(\alpha \in[-\beta, \beta])$. 1 .

(a) The set $\Omega \sim \cup_{\alpha \in(-\beta, \beta)} \Omega_{\alpha}$ contains a residual subset of $\Omega$, and has $\mu_{0}$-measure

(b) The set $\Omega \sim \cup_{\alpha \in[-\beta, \beta]} \Omega_{\alpha}$ contains a residual set in $\Omega$.

Proof. (a) Let $\Omega_{1} \subset \Omega$ be the set discussed in 3.4, and let $\Omega_{2} \subset \Omega$ be the set discussed in 3.5. Then $\mu_{0}\left(\Omega_{1}\right)=1$, and $\Omega_{2}$ is a residual subset of $\Omega$. Choose $\alpha$ such that $-\beta<\alpha<\beta$. Then $\Omega_{\alpha} \cap \Omega_{1}=\varnothing$, and $\Omega_{\alpha} \cap \Omega_{2}=\varnothing$. For, suppose, e.g., that $\omega \in \Omega_{\alpha} \cap \Omega_{1}$. Let $x_{1}(t)$ be a solution of $(1)_{\omega}$ satisfying $\lim _{|t| \rightarrow \infty}(1 / t) \ln \left\|x_{1}(t)\right\|=\beta$, and let $x_{\alpha}(t)$ be a solution of (1) satisfying $e^{-\alpha t}\left\|x_{\alpha}(t)\right\|<C<\infty$ for all $t$. If $\Psi(t)$ is the fundamental matrix solution of $(1)_{\omega}$ whose columns are $x_{1}(t)$ and $x_{\alpha}(t)$, then 
$\lim _{t \rightarrow-\infty} \operatorname{det} \Psi(t)=0$. However, $\operatorname{tr} b(\omega)=0$ and Liouville's formula imply that $\operatorname{det} \Psi(t)=$ const $\neq 0$. Hence $\Omega_{\alpha} \cap \Omega_{1}$ must be empty. Similarly, $\Omega_{\alpha} \cap \Omega_{2}=\varnothing$.

(b) We will show only that $\Omega \sim \Omega_{\beta}$ contains a residual set; the proof for $\Omega \sim \Omega_{-\beta}$ is similar. By the proof of [3, Theorem 4.3], the minimal subset $M$ of $\Sigma$ contains a residual subset $M_{3}$ such that

$$
(\omega, \varphi) \in M_{3} \Rightarrow\left(\lim _{t \rightarrow \infty}, \varlimsup_{t \rightarrow \infty}\right)\left[\beta t+\int_{0}^{t} f_{*}((\omega, \varphi) \cdot s) d s\right]=(-\infty, \infty) .
$$

By [11, Lemma 3.1], $\pi_{p}\left(M_{3}\right)=\Omega_{3}$ is a residual subset of $\Omega$. Let $(\omega, \varphi) \in M_{3}$, and let $x(t)$ be a solution to $(1)_{\omega}$ such that $x(0) \neq 0$ lies on the line in $\mathbf{R}^{2}$ determined by $\varphi$. Then

$$
\left(\lim _{t \rightarrow \infty}, \varlimsup_{t \rightarrow \infty}\right) e^{-\beta t}\|x(t)\|=(0, \infty) .
$$

Now, if $\omega$ were in $\Omega_{\beta}$ as well as in $\Omega_{3}$, then (1) would have a solution $x_{\beta}(t)$ satisfying $\left\|x_{\beta}(t)\right\| \leqslant C e^{-\beta t}$ for some constant $C(-\infty<t<\infty)$. Then $x(t)$ and $x_{\beta}(t)$ would be linearly independent. A fundamental matrix argument like that used in part (a) would yield a contradiction. We conclude that $\Omega \sim \Omega_{\beta}$ contains the residual set $\Omega_{3}$.

\section{REFERENCES}

1. R. Ellis, Lectures on topological dynamics, Benjamin, New York, 1969.

2. R. Johnson, Ergodic theory and linear differential equations, J. Differential Equations 28 (1978), 23-34.

3. __ Minimal functions with unbounded integral, Israel J. Math. 31 (1978), 133-141.

4. V. M. Millionšcikov, Proof of the existence of irregular systems of linear differential equations with almost periodic coefficients, Differential Equations 4 (1968), 203-205.

5.,$A$ proof of the existence of nonregular systems of linear differential equations with quasiperiodic coefficients, Differential Equations 5 (1969), 1475-1478.

6. Statistically regular systems, Math. USSR-Sb. 4 (1968), 125-135.

7. V. V. Nemytski and V. V. Stepanov, Qualitative theory of differential equations, English translation, Princeton Math. Series, no. 22, Princeton Univ. Press, Princeton, N. J., 1960.

8. R. J. Sacker and G. R. Sell, Existence of the dichotomies and invariant splittings for linear differential systems. I, J. Differential Equations 15 (1974), 429-458.

9. $320-358$.

10. G. R. Sell, Linear differential systems, Lecture notes, University of Minnesota, 1975.

11. W. A. Veech, Point-distal flows, Amer. J. Math. 92 (1970), 205-242.

12. R. E. Vinograd, A problem suggested by N. P. Erugin, Differential Equations 11 (1975), 474-478.

Department of Mathematics, University of Southern California, los Angeles, California 90007 